\title{
Cryptocurrencies and Blockchains
}

Quinn DuPont, Cryptocurrencies and Blockchains. Polity Press: Cambridge, 2019; 224 pp,;ISBN 9781509520237, £15.99 (pbk)

Reviewed by: Tom Redshaw, University of Salford, UK.

Blockchain technology is notoriously nebulous. Not only is its functionality difficult to define, it is a technology shrouded by discourses of hyperbole, utopia, and criminality. Blockchain technology is at the same time heralded as a revolution in finance; the basis for numerous utopian projects; and a key infrastructure for crime in the digital age. ${ }^{i}$ Demystifying blockchain technologies therefore, and shedding light on how they function as global networks of people and machines, is a crucial task. In this respect, Quinn DuPont's Cryptocurrencies and Blockchains presents the most clear and comprehensive contribution to date.

First, to understand what blockchain technology is and why it is an important topic for analysis, it is necessary to break it down into its various forms and their functionalities, their respective histories and their social impact across the world. The first and still most widely used variant of blockchain technology is the cryptocurrency Bitcoin. Bitcoin has a fascinating and mysterious history as the culmination of several attempts to develop an electronic cash system, a currency for internet users to exchange outside the purview of financial institutions and regulatory bodies. DuPont tells this story, but unlike others in the field such as Golumbia (2016) and Brunton (2019), DuPont foregrounds the technical innovations made and their capacity to serve a number of functions. This is preceded by a concise list of definitions for the terminology involved, and is followed by a discussion of the narratives that have emerged among many users for whom Bitcoin is a revolutionary form of money. By foregrounding the technical elements, DuPont is able to differentiate between the particular constellation that comes together as Bitcoin and its many imitations that function as money - cryptocurrencies; and the more recent experimentations with the technology that go beyond money and provide a basis for storing data across decentralised networks - blockchains. In both cases, DuPont asserts that the underlying technology is social as it coordinates human activities and carries meanings between social groups. In this sense, DuPont argues, blockchain technology can be better understood as media.

Defining blockchain technologies as media is DuPont's major theoretical contribution. Cryptocurrencies are examined in this light, advancing theories of money as media developed by Georg Simmel and initially applied to Bitcoin by Nigel Dodd (2018). When DuPont turns this lens to blockchains more broadly, he offers a novel and incisive approach for interpreting new sociotechnical phenomena. Drawing on several media theorists and actor-network scholars, DuPont outlines two ways in which blockchains function as media. First, blockchains are media in the classic sense that they 'sit between people, carry meaning, and transform messages' (p. 82). This is why the technology provides more than a system for exchanging digital tokens - in principle, the information 
stored and exchanged could pertain to anything, not just transaction records. Second, blockchains are media in the sense that they provide abstract representations that stand in for, and give meaning to, complex processes. The prime example for this that DuPont gives is the peculiar hashing function at the heart of most blockchain technologies. This function is a highly complex cryptographic process but one that produces, in the case of Bitcoin, a unit of digital currency - something that is held to have value and meaning by social groups. Again, this need not be digital currency, it could be a unique representation of anything and this is where the use value of the technology lies: its 'ability to abstract complexity and make objects discrete' (p. 83). This is DuPont's focus as he moves through various experimentations with blockchains in different contexts.

There are two major contexts in which blockchain technology has been adapted and developed in recent years: markets and law. For the former, this relates mostly to criminal markets and financial markets. For the latter, blockchain technology is explored both in terms of how it can be successfully regulated and also how it can serve to regulate. The role of Bitcoin and other cryptocurrencies in facilitating the rise of markets for illicit goods online is considerable and has led to its own field of investigation into 'cryptomarkets' and the 'dark web' (Cunliffe et al., 2019). DuPont's focus, however, rests mostly with financial markets, where blockchain technology is being developed not only as the basis for new and increasingly widespread practices in cryptocurrency trading, but also as an infrastructure for assisting the clearance and settlement processes undertaken by major financial institutions. Here, DuPont draws on several interviews with industry leaders, as well as recounting his own experiences experimenting with the technology, to offer a cautious and balanced appraisal of the potential for blockchains to transform finance, providing an antedote to the hype that often circulates among 'fintech' entrepreneurs.

Law is perhaps the most significant context for examining blockchain technology moving forward. From the early days of Bitcoin's emergence, scholars such as De Filippi and Hassan (2016) were assessing how blockchain not only presented considerable obstacles for regulators but also possibilities for coordinating human behaviour, possibilities increasingly referred to as algorithmic governance. DuPont's final three chapters examine blockchain in this context, detailing how various innovations such as 'smart contracts' and 'smart property' present challenges to regulators, and also how blockchains may play a central role in the coordination of key new sectors such as 'smart manufacturing' and the 'internet of things'. This concludes with an analysis of the most ambitious development in blockchain technology, the attempt to build 'decentralised autonomous organisations' (DAOs): an entirely new kind of organisation where the coordination of labour, the management of assets and exchanges of information are all automated and executed by machines following particular sets of rules. DuPont presents a clear discussion of this most complex of topics in his final chapter, noting the serious challenges this presents (e.g. if algorithms govern, who governs the algorithms?) as well as the possibilities. Researching these possibilities even led DuPont to attempt to establish his own DAO which would give whales the power to prosecute humans for oil spills, yet in the end 
his platform was hacked and shut down, a story that perhaps encapsulates perfectly why blockchain technology remains shrouded by hyperbole, utopianism and criminality.

References

Brunton, F. (2019) Digital Cash. Oxford: Princeton University Press

Cunliffe, J. Martin, J. \& Munksgaard, R. (2019) Cryptomarkets: A Research Companion. Bingley: Emerald

De Filippi, P, Hassan, S (2016) Blockchain technology as a regulatory technology: from code is law to law is code. First Monday 21(12).

DOI: $10.5210 / \mathrm{fm} . v 21 \mathrm{i} 12.7113$.

Dodd, Nigel. (2018). “The Social Life of Bitcoin”. Theory, Culture, \& Society. 35(3): 35-56.

Golumbia, D. (2016) "Politics of Bitcoin: Software as Right-wing Extremism". Minneapolis, MN: University of Minnesota Press.

Tapscott, D. \& Tapscott, A. (2016) Blockchain Revolution. London: Penguin Books.

i For one of many examples of blockchain technology as a revolution in finance, see Tapscott, D. \& Tapscott, A. (2016); for the role of blockchain in utopian projects see Brunton (2019); for criminality, Cunliffe et al (2019). 